\title{
Flavonoids and Diarylheptanoids: Neuroprotective Activities of Phytochemicals
}

\author{
Kathyani Parasram ${ }^{1, a^{*}}$ \\ ${ }^{1}$ Department of Biology, University of Windsor, 401 Sunset Ave., Windsor, ON, Canada, N9B 3P4 \\ a * parasra@uwindsor.ca
}

Keywords: flavonoids, diarylheptanoids, phenolic compounds, neuroscience, drug design, phytochemicals, neurodegenerative diseases, Alzheimer's disease, Parkinson's disease.

\begin{abstract}
Plants are often used as sources of lead compounds with phenolic compounds frequently attributed to physiological effects. Flavonoids and diarylheptanoids are important groups of phenolic compounds that impart antioxidant, antitumorgenic, antinflammatory, and neuroprotective effects. These neuroprotective effects can be harnessed to develop treatments for neurodegenerative diseases, such as Alzheimer's and Parkinson's disease. Recent discoveries have characterized new neuroprotective compounds and/or sources and tested treatments on cell lines and model animals to improve treatments for future persons with neurodegenerative disorders.
\end{abstract}

\section{Introduction}

The use of phytochemicals as a source of lead compounds in pharmaceutical drug discovery is well known $[1,2]$. These phytochemicals are often extracts from plants used in traditional medicine and may be independent active ingredients or natural formulations that can be used to enhance the desired effects of drugs $[3,1]$. The biological activity of plants may be due to phenolic compounds that are known to confer antioxidant, antitumorgenic, antinflammatory, or other favourable activities [3-5]. One of the many potential pharmaceutical uses of phenolic compounds is the treatment and prevention of neural diseases [6-8]. Phenolic compounds that can cross the bloodbrain barrier can indicate neuroprotective functions and clinical potential of the central nervous system which has a limited ability to repair and regenerate [3, 9, 10]. Two major groups of neuroprotective phytochemicals are diarylheptanoids, such as curcumin, and flavonoids, such as quercetin (Fig. 1) [6, 11]. Flavonoids and diarylheptanoids are neuroprotective, are able to protect neurons from damage, particularly through free radical scavenging activity, modulation of neuroplasticity resulting in an increase in cognitive, behavioral and/or emotional function, e.g., improving memory $[11,6,12]$. These compounds may serve as active ingredients for novel therapies in the treatment of neurodegenerative diseases including Alzheimer's and Parkinson's disease [6].

(a)

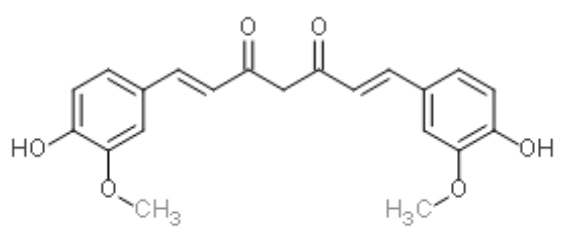

(b)

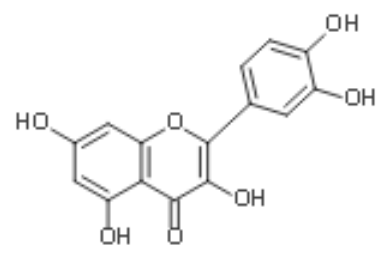

Figure 1. Structure of Curcumin (a) and Quercetin (b) [13-15].

Neurodegenerative diseases, such as Alzheimer's disease, Parkinson's disease, ALS, and Huntington's disease, are characterized by memory loss, irregular movement or behaviour and emotional difficulties due to a loss of neurons in the central nervous system $[1,16]$. Alzheimer's disease is a neurodegenerative disease in which beta-amyloid accumulates causing loss of memory which may result in dementia [11]. Parkinson's disease is a result of loss of dopaminergic receptor function, thereby reducing dopamine and causing symptoms, such as resting tremors and rigid limbs 
[17]. These neurodegenerative disorders may be caused by a combination of environmental, genetic, and age-related factors and can be treated using conventional drug therapies which are currently only moderately effective and often focus on symptom control not reversal or prevention of progression [16, 8]. Oxidative stress is often a factor in neurodegenerative disease development and progression and can be remedied using antioxidant drugs, or natural compounds i.e., phenolic compounds including flavonoids and diarylheptanoids [3, 1, 18]. This paper will outline recent discoveries of flavonoids and diarylheptanoids for the treatment of neurodegenerative diseases.

\section{Neurodegenerative Diseases}

Quercetin and curcumin are two of the most well-known anti-inflammatory, antioxidant and consequently neuroprotective natural compounds [3]. Quercetin research shows potential for the treatment of Alzheimer's disease, as demonstrated in a mice model which administered quercetin intraperitoneally every 48 hours for 3 months resulted in decrease in beta-amyloidosis and tauopathy in the hippocampus and amygdala and an increase in cognitive performance although it may be toxic at high (greater than $100 \mu \mathrm{M})$ concentrations $[11,19]$. Curcumin has attracted a lot of attention due to its potential for both cancer and neurodegenerative disease treatment by reducing the damage due to oxidative stress in the body [20-22]. Research has shown that curcumin increases hippocampal neurogenesis in rats thereby preventing and potentially reversing cellular effects of neurodegenerative diseases [20-22]. From a pharmaceutical view one of the major hurdles with curcumin stems from its low solubility and low retention time in the body, these hurdles are being overcome by innovative delivery systems such as the PLGA nanoparticles which one group found increases half-time in the cerebral cortex and hippocampus by more than 1.5 fold [22]. Building upon the success of Quercetin and Curcumin for the treatment of neurodegenerative diseases, other flavonoids and diarylheptanoids have been studied for pharmaceutical potential.

\section{Alzheimer's}

Investigations into specific activity of these molecules on Alzheimer's disease models were conducted in order to assess disease-specific potential of compounds [1, 8, 23]. Nobiletin from citrus was tested at a $30 \mathrm{mg} / \mathrm{kg}$ dose over three months in a triple transgenic mouse model and was found to reverse the short-term memory and recognition task performance by reducing reactive oxygen species in the hippocampus [1]. In humans a small group of Australian adults over age 65 with Alzheimer's related dementia showed an increase in cognitive functions when their diet had a higher total flavonoid content [24].

\section{Parkinson's}

The effect of flavonoids (flavonoid glycosides and quinochalcones) from safflower extract increased PC12 cell viability and behavioral performance of 6-OHDA (Parkinson's model) rats [12]. The use of flavonoids, such as naringin, to suppress microglial activation in animal models of Parkinson's disease was tested and showed neuroprotective behaviour in the substantia nigrapars compacta in the rat brain. However, at high levels of inflammation the neuroprotective flavonoids generated neurotoxic compounds [17].

\section{Neuroprotective Phytochemical Studies}

The neuroprotective effects of flavonoids and diarylheptanoids were tested using H202 and glutamate stressed cell lines [18, 14, 25-31]. This test mimics oxidative stress that is attributed to the development of neurodegenerative disorders [18]. Girsium setidens, Aster scaber, Passiflora actinia, Ginko Biloba, Acel nikoense, Alnus glutinosa, Alpinia officinavum Hance, Panax ginseng, Schisandra chinensis, Rehmannia glutinosa, Flammulina velutipes, Rhododendron fortune, Morus alba, and Carya cathayensis Sarg. were all recently shown to contain flavonoids and/or diarylheptanoids that could counteract the neurodegenerative effects of oxidative stress $[18,25,9,13,28-35]$. The ability of flavonoids to cross the blood-brain barrier was enhanced by 
alpha-tocopherol as verified by the increase in the presence of flavonoid derivatives in the brain and plasma as detected by high performance liquid chromatography mass spectrometry [14]. The use of rutin on rats in $100 \mathrm{mg} / \mathrm{kg} /$ day dosage for five weeks suggests an application for rutin in the treatment of the neurodegenerative eye disease diabetic retinopathy since rutin promotes nerve growth [36]. While flavonoids and diarylheptanoids were found to activate the PI3/Akt, MAPK, PKA, CHOP pathways and Nrf2 concentration, they did not affect Ca2+ homeostasis [28, 27, 3739]. A potential application of neuroprotective flavonoids and diarylheptanoids is in the protection of the brain from damage caused by ischemic stroke as demonstrated by the ability of flavonoids to regulate blood pressure and serum lipid levels [26].

\section{Conclusion}

The search for cures to neurodegenerative diseases is well rooted in phytochemical analysis. The antioxidative role of flavonoids and diarylheptanoids plays an important role in the hunt for effective treatments and prevention of disease. In particular, research has focused on Alzheimer's disease and Parkinson's disease treatments by flavonoids and diarylheptanoids due to the deficiencies in current treatments. Diarylheptanoids and flavonoids provide new groups of compounds that can be used to develop novel classes of drug treatments for neurodegenerative diseases that target disease mechanisms. Further research into the pharmacokinetics and phamacodynamics of phenolics may lead to the development of biologically available and highly bioactive drugs.

\section{References}

[1] A. Nakajima et al., Nobiletin, a citrus flavonoid, improves cognitive impairment and reduces soluble $A \beta$ levels in a triple transgenic mouse model of Alzheimer's disease (3XTg-AD), Behav. Brain Res. 289 (2015) 69-77.

[2] S.L. Costa et al., Impact of plant-derived flavonoids on neurodegenerative diseases, Neurotox. Res. (2016) 1-12.

[3] F. Moosavi et al., Modulation of neurotrophic signaling pathways by polyphenols, Drug Des. Develop. and Therapy. 10 (2016) 23.

[4] I. Solanki et al., Flavonoid-based therapies in the early management of neurodegenerative diseases, Advances in Nutrition: An International Review Journal. 6(1) (2015) 64-72.

[5] J. Xu, M.H. Lacoske, E.A. Theodoraki, Neurotrophic natural products: chemistry and biology, Angewandte Chemie Int. Ed. 53(4) (2014) 956-987.

[6] G. Tang et al., A natural diarylheptanoid promotes neuronal differentiation via activating ERK and PI3K-AKT dependent pathways, Neurosci. 303 (2015) 389-401.

[7] C. Rendeiro, J.S. Rhodes, J.P. Spencer, The mechanisms of action of flavonoids in the brain: direct versus indirect effects, Neurochem. Int. 89 (2015) 126-139.

[8] S.L. Xu et al., Flavonoids, derived from traditional Chinese medicines, show roles in the differentiation of neurons: possible targets in developing health food products, Birth Defects Res. Part C: Embryo Today: Rev. 99(4) (2013) 292-299.

[9] Y. Wu et al., Study of neuroprotective function of Ginkgo biloba extract (EGb761) derived-flavonoid monomers using a three-dimensional stem cell-derived neural model, Biotech. Progress. 32(3) (2016) 735-744.

[10] G.A.R. Johnston, Flavonoid nutraceuticals and ionotropic receptors for the inhibitory neurotransmitter GABA, Neurochem. Int. 89 (2015) 120-125. 
[11] A.M. Sabogal-Guáqueta et al., The flavonoid quercetin ameliorates Alzheimer's disease pathology and protects cognitive and emotional function in aged triple transgenic Alzheimer's disease model mice, Neuropharmaco. 93 (2015) 134-145.

[12] R. Ren et al., Neuroprotective Effects of a standardized flavonoid extract of safflower against neurotoxin-induced cellular and animal models of Parkinson's disease, Sci. Reports. 6 (2016).

[13] J. Dinić et al., Chemo-protective and regenerative effects of diarylheptanoids from the bark of black alder (Alnus glutinosa) in human normal keratinocytes, Fitoterapia. 105 (2015) 169-176.

[14] P. Ferri et al., Enhancement of flavonoid ability to cross the blood-brain barrier of rats by coadministration with $\alpha$-tocopherol, Food and Func. 6(2) (2015) 394-400.

[15] Information on https://web.chemdoodle.com/demos/sketcher/.

[16] I. Solanki, P. Parihar, M.S. Parihar, Neurodegenerative diseases: From available treatments to prospective herbal therapy, Neurochem. Int. 95 (2016) 100-108.

[17] S.R. Kim, Inhibition of microglial activation and induction of neurotrophic factors by flavonoids: a potential therapeutic strategy against Parkinson's disease, Neural Regen. Res. 10(3) (2015) 363.

[18] M.J. Chung et al., Neuroprotective effects of phytosterols and flavonoids from Cirsium setidens and Aster scaber in human brain neuroblastoma SK-N-SH cells, Life Sci. 148 (2016) 173-182.

[19] F. Dajas et al., Quercetin in brain diseases: Potential and limits, Neurochem. Int. 89 (2015) 140-148.

[20] Y. Xu et al., Curcumin reverses impaired hippocampal neurogenesis and increases serotonin receptor 1A mRNA and brain-derived neurotrophic factor expression in chronically stressed rats, Brain Res. 1162 (2007) 9-18.

[21] Y.M. Tsai et al., Curcumin and its nano-formulation: the kinetics of tissue distribution and blood-brain barrier penetration, Int. J. Pharmaceutics. 416(1) (2011) 331-338.

[22] S. Dong et al., Curcumin enhances neurogenesis and cognition in aged rats: implications for transcriptional interactions related to growth and synaptic plasticity, PLoS One. 7(2) (2012) e31211.

[23] N. Cho et al., Ameliorative effect of betulin from Betula platyphylla bark on scopolamineinduced amnesic mice, Biosci. Biotech. Biochem. 80(1) (2016) 166-171.

[24] K. Caldwell et al., Dietary flavonoid intake and cognitive performance in older adults with Alzheimer's type dementia, J. of Aging Res. and Clinical Practice. 5(2) (2016) 93-97.

[25] K.C. Santos et al., Passiflora actinia hydroalcoholic extract and its major constituent, isovitexin, are neuroprotective against glutamate-induced cell damage in mice hippocampal slices, J. Pharm. Pharmacology. 68(2) (2016) 282-291.

[26] M.I. Ayuso, J. Montaner, Advanced neuroprotection for brain ischemia: an alternative approach to minimize stroke damage, Expert Opinion on Investig. Drugs. 24(9) (2015) 1137 1142.

[27] C. Echeverry et al., Antioxidant activity, cellular bioavailability, and iron and calcium management of neuroprotective and nonneuroprotective flavones, Neurotox. Res. 27(1) (2015) 31-42.

[28] D.S. Lee et al., Acerogenin A from Acer nikoense maxim prevents oxidative stress-induced neuronal cell death through Nrf2-mediated heme oxygenase-1 expression in mouse hippocampal HT22 cell line, Molecules. 20(7) (2015) 12545-12557.

[29] Q. Hu et al., Identification of flavonoids from Flammulina velutipes and its neuroprotective effect on pheochromocytoma-12 cells, Food Chem. 204 (2016) 274-282. 
[30] Y. Lai et al., 6, 8-Di-C-methyl-flavonoids with neuroprotective activities from Rhododendron fortune, Fitoterapia. 112 (2016) 237-243.

[31] J.W Jung et al., Isoprenylated flavonoids from the root bark of Morus alba and their hepatoprotective and neuroprotective activities, Archives of Pharmacal Res. 38(11) (2015) 2066-2075.

[32] L.P. Köse et al., LC-MS/MS analysis, antioxidant and anticholinergic properties of galanga (Alpinia officinarum Hance) rhizomes, Indust. Crops and Prod. 74 (2015) 712-721.

[33] J. Li et al., Natural therapeutic agents for neurodegenerative diseases from a traditional herbal medicine Pongamia pinnata (L.) Pierre, Bioorganic and Med. Chem. Letters. 25(1) (2015) 5358.

[34] M.E. Pak et al., Studies on medicinal herbs for cognitive enhancement based on the text mining of Dongeuibogam and preliminary evaluation of its effects, J. Ethnopharmacol. 179 (2016) 383-390.

[35] L. Xiang et al., Chemical constituent and antioxidant activity of the husk of Chinese hickory, J. Funct. Foods. 23 (2016) 378-388.

[36] M.S. Ola et al., Neuroprotective effects of rutin in streptozotocin-induced diabetic rat retina, J. Mol. Neurosci. 56(2) (2015) 440-448.

[37] S.D. Wu et al., Ginsenoside-Rd promotes neurite outgrowth of PC12 cells through MAPK/ERK-and PI3K/AKT-dependent pathways, Int. J. Mol. Sci. 17(2) (2016) 177.

[38] J.L. Zhen et al., Luteolin rescues pentylenetetrazole-induced cognitive impairment in epileptic rats by reducing oxidative stress and activating PKA/CREB/BDNF signaling, Epilepsy and Behavior. 57 (2016) 177-184.

[39] Q. Zhao et al., Echinacoside protects against MPP+-induced neuronal apoptosis via ROS/ATF3/CHOP pathway regulation, Neurosci. Bulletin. 32(4) (2016) 349-362. 